\title{
OPTIMUM vS. CORRELATION METHODS IN TRACKING RANDOM SIGNALS IN BACKGROUND NOISE*
}

\author{
BY \\ R. C. DAVIS** \\ Hughes Tool Company, Aircraft Division, Culver City, California
}

I. Summary. A method of target tracking used exclusively in some applications is that of space diversity reception. We consider a two-dimensional problem with two receivers, although in principle the techniques developed are applicable to a threedimensional problem utilizing three or more receivers. With $N$ receivers the computational difficulties increase as $N^{2}$. The tracking problem considered here is formulated as follows. During a time interval $0 \leq t \leq T$ one observes random processes $x_{1}(t)=$ $s(t)+n_{1}(t)$ and $x_{2}(t)=s\left(t+\tau_{0}\right)+n_{2}(t)$, and one desires to estimate the unknown value of $\tau_{0} . s(t), n_{1}(t)$, and $n_{2}(t)$ are continuous, Gaussian, stationary processes with continuous, monotonoid covariance functions and with $E s(t) n_{1}\left(t^{\prime}\right)=E s(t) n_{2}\left(t^{\prime}\right)=0$. $n_{1}(t)$ and $n_{2}(t)$ are stationarily cross-correlated with a cross-correlation function that is in general an asymmetric function of time delay. As a criterion for an optimum estimate, $\tau_{0}^{\prime}$, of $\tau_{0}$, we use the one-commonly used in statistical literature, that $\tau_{0}^{\prime}$ should have minimum variance about the true value $\tau_{0}$; that is, $E\left(\tau_{0}^{\prime}-\tau_{0}\right)^{2}$ be a minimum. It is shown that for small values of $\omega_{u} \tau_{0}$ (where $\omega_{u}$ is the highest frequency in the tracking pass band) the normalized error variance $E\left[\left(\tau_{0}^{\prime}-\tau_{0}\right) / \tau_{0}\right]^{2}$ is identical to the reciprocal of the output signal-to-noise ratio- the criterion of common use in engineering literature on correlation methods. Using the Cramér-Rao inequality, an explicit expression for the minimum variance attainable by any estimate whatsoever is established. Moreover we arrive at the interesting conclusion that even with the use of optimum pre-detection filtering in a correlation system, the variance of the correlator estimate of $\tau_{0}$ is always greater than the minimum variance given by the Cramer-Rao inequality. Gains in output signal-to-noise ratio obtained by an optimum system over a correlator vary in accordance with the statistical properties of signal and noise backgrounds. In general the gains are greater with asymmetrical cross-correlation between $n_{1}(t)$ and $n_{2}(t)$ than otherwise. From a practical viewpoint gains obtainable over correlation methods become important for large values of the time-bandwidth product. This is due to the fact that under this condition the maximum likelihood estimate of $\tau_{0}$ has an error variance that is approximately equal to the minimum variance obtainable by any estimate. For small values of the time-bandwidth product no theory exists about the variance of the maximum likelihood estimate, since no efficient estimate of $\tau_{0}$ exists. For this case, a worthwhile experimental study would be to compare the variance of the maximum likelihood estimate with the minimum variance calculated from the Cramér-Rao inequality.

Finally it is shown how the maximum likelihood estimate is constructed and noted that the construction involves a considerable number of operations performed simultaneously during the time interval $T$. In order to simplify the system, an approximate maximum likelihood estimate is obtained that is optimum only "on target", and its properties are discussed.

\footnotetext{
*Received March 6, 1956.

** Now employed by Engineering Department of Convatir, Ceneral 1) Mnimices Corporation, Pomona, Cal.
} 
II. Introduction. Heretofore problems involving several Gaussian stationary processes (such as the one outlined above) have been approached by the use of correlation methods that have been used extensively in engineering literature. With these methods the goal is the natural one of maximizing the output signal-to-noise ratio. On the other hand there is a voluminous statistical literature on methods of analyzing problems of estimation of unknown parameters of probability distributions. The purpose of this paper is to apply some of these methods to the problem outlined. Using the same criterion of optimum as the one used in correlation methods, our method is more powerful. This is to be expected, since the best statistical techniques make use of the joint probability distribution of the coordinates of the various random processes entering into the problem. On the other hand correlation methods make use only of one second moment of the joint probability distribution. Since gains are demonstrated for Gaussian distributions (using all second moments in a particular combination), the author surmises that in problems involving non-Gaussian processes very large gains over correlation methods are to be expected. Unfortunately these gains are not obtained without difficulties. The main reason that modern statistical techniques have not been used extensively in noise problems is the formidable difficulty in obtaining coordinate systems that have the property that any finite number of coordinates are stochastically independent. In the case of Gaussian processes the central difficulty is that of matrix inversion. In problems involving a single random process, an orthogonal decomposition is possible by a method described by Karhunen [1]. In the case of two random processes (uncorrelated or not) possessing different power spectrum shapes, there exists no single set of orthogonal eigenfunctions that simultaneously yield orthogonal decompositions of both random processes. However, as we show later, in calculating the lower bound given by the Cramerr-Rao inequality, we are able to avoid this difficulty. In order to obtain an explicit expression for the maximum likelihood estimate, however, we are forced to use much stronger assumptions on the statistical characteristics of signal and noises.

III. Derivation of the Cramerr-Rao lower bound. Consider the following problem. During the time interval $0 \leq t \leq T$ we observe the random processes $x_{1}(t)=s(t)+$ $n_{1}(t)$ and $x_{2}(t)=s\left(t+\tau_{0}\right)+n_{2}(t) . \tau_{0}$ is a fixed but unknown parameter. The problem is to determine the minimum variance of any unbiased estimate of $\tau_{0}$. We make the following assumptions concerning the characteristics of $s(t), n_{1}(t)$, and $n_{2}(t)$.

Assumption 1. $s(t), n_{1}(t)$, and $n_{2}(t)$ are continuous, stationary, Gaussian processes, each with zero mean value, finite variance, and each possessing a monotonoid and continuous covariance function.

Assumption 2. $s(t)$ is uncorrelated with $n_{1}(t)$ and also with $n_{2}(t)$.

Assumption 3. $n_{1}(t)$ and $n_{2}(t)$ are stationarily cross-correlated with a monotonoid and continuous cross-covariance function.

It follows from Assumption 3 and a result due to Cramér [2, p. 227] that if we write

$$
\rho_{12}(\tau)=E n_{1}(t) n_{2}(t+\tau),
$$

then

$$
\rho_{12}(\tau)=\int_{0}^{\infty}\left[\cos \omega \tau d \phi_{12}(\omega)+\sin \omega \tau d \psi_{12}(\omega)\right] .
$$

The spectral functions $\phi_{12}(\omega)$ and $\psi_{12}(\omega)$ are of bounded variation in $(0, \infty)$. Each function is the sum of an absolutely continuous function and a discontinuous function. 
(corresponding to possible discrete line frequencies in the spectrum). If no lines exist in the cross spectrum, (3.1) reduces to

$$
\rho_{12}(\tau)=\int_{0}^{\infty}\left[\phi_{12}(\omega) \cos \omega \tau+\psi_{12}(\omega) \sin \omega \tau\right] d \omega .
$$

Similarly we denote by $\phi_{0}(\omega), \phi_{1}(\omega)$, and $\phi_{2}(\omega)$ the power spectra of $s(t), n_{1}(t)$, and $n_{2}(t)$ respectively. Each power spectrum is related to its corresponding covariance function, $\rho(\tau)$, by the Wiener-Khintchine relation

$$
\rho(\tau)=\int_{0}^{\infty} \cos \omega \tau d \phi(\omega)
$$

where $\phi(\omega)$ is of bounded variation in $(0, \infty)$. The first step consists in finding coordinate systems for $x_{1}(t)$ and $x_{2}(t)$ so that we can obtain an expression for the probability of realizing a given combination of $x_{1}(t)$ and $x_{2}(t)$. Since any practical system possesses a finite time-bandwidth product, we shall see that we require only a finite number of coordinates. To obtain the coordinates we use a slight extension of a method given by Root and Pitcher [3, p. 314]. It follows from Assumption 1 that we can expand $\rho_{c}(\tau)$ in a Fourier series valid in the interval $-T \leq \tau \leq T$ [see 4, Chap. 6]. This gives

$$
\rho_{s}(\tau)=\sum_{K=1}^{\infty} \theta_{K} \cos \omega_{0} K \tau,
$$

where $\omega_{0}=\pi / T$. Then there exists a process $S(t)$ defined by

$$
S(t)=\underset{n \rightarrow \infty}{\lim } \sum_{K=1}^{n}\left[a_{K} \cos \omega_{0} K t+b_{K} \sin \omega_{0} K t\right]
$$

where the $a_{K}, b_{K}$ are Gaussian variables satisfying

$$
\begin{gathered}
E a_{K}=E b_{K}=0, \quad \text { for } \quad K=1,2, \cdots \\
E a_{j} a_{K}=E b_{i} b_{K}=\delta j_{K} \theta_{K}, \quad E a_{i} b_{K}=0, \quad j=1,2, \cdots, \quad K=1, \overline{2}, \cdots,
\end{gathered}
$$

and the process $S(t)$ has the same multi-variate distributions as $s(t)$. (This follows from the easily verified fact that $E S(t) S(t+\tau)=E s(t) s(t+\tau)$ for every $\tau \leq T$.) Similarly, for the processes $n_{1}(t)$ and $n_{2}(t)$ we write

$$
\begin{aligned}
\rho_{1}(\tau) & =\sum_{K=1}^{\infty} \alpha_{K} \cos \omega_{0} K \tau, \\
\rho_{2}(\tau) & =\sum_{K=1} \beta_{K} \cos \omega_{0} K \tau \\
\rho_{12}(\tau) & =\sum_{K=1}\left[\gamma_{K} \cos \omega_{0} K \tau+\epsilon_{K} \sin \omega_{0} K \tau\right]
\end{aligned}
$$

for $-T \leq \tau \leq T$, and correspondingly there exist processes $N_{1}(t)$ and $N_{2}(t)$ possessing the same joint multi-variate distributions as $n_{1}(t)$ and $n_{2}(t)$. We define

$$
\begin{aligned}
& N_{1}(t)=\underset{n \rightarrow \infty}{\operatorname{li} . \mathrm{m}} \cdot \sum_{K=1}^{n}\left[u_{K} \cos \omega_{0} K t+v_{K} \sin \omega_{0} K t\right], \\
& N_{2}(t)=\underset{n \rightarrow \infty}{\text { l.i.m. }} \sum_{K=1}^{n}\left[p_{K} \cos \omega_{0} K t+q_{K} \sin \omega_{0} K t\right],
\end{aligned}
$$


for $0 \leq t \leq T$. Then we have

$$
\begin{gathered}
E u_{K}^{2}=E v_{K}^{2}=\alpha_{K}, \quad E p_{K}^{2}=E q_{K}^{2}=\beta_{K}, \\
E u_{K} p_{K}=E v_{K} q_{K}=\gamma_{K}, \quad E u_{K} q_{K}=-E v_{K} p_{K}=\epsilon_{K} \\
\text { for } K=1,2, \cdots .
\end{gathered}
$$

Moreover, every amplitude at frequency $j \pi / T$ is uncorrelated with every amplitude at frequency $K \pi / T$ for $j \neq K$.

From the above it follows that there exist Gaussian processes $X_{1}(t)$ and $X_{2}(t)$ which possess the same joint multi-variate distributions as $x_{1}(t)$ and $x_{2}(t)$. Clearly

$$
\begin{aligned}
& X_{1}(t)=S(t)+N_{1}(t)=\underset{n \rightarrow \infty}{\text { li.m. }} \sum_{K=1}^{n}\left[A_{K} \cos \omega_{0} K t+B_{K} \sin \omega_{0} K t\right], \\
& X_{2}(t)=S\left(t+\tau_{0}\right)+N_{2}(t)=\underset{n \rightarrow \infty}{\text { l.i.m. }} \sum_{K=1}^{n}\left[C_{K} \cos \omega_{0} K t+D_{K} \sin \omega_{0} K t\right],
\end{aligned}
$$

for $0 \leq t \leq T$. We have

$$
\begin{array}{ll}
A_{K}=a_{K}+u_{K}, & E A_{K}^{2}=\theta_{K}+\alpha_{K}, \\
B_{K}=b_{K}+v_{K}, & E B_{K}^{2}=\theta_{K}+\alpha_{K}, \\
C_{K}=a_{K} \cos \omega_{0} K \tau_{0}+b_{K} \sin \omega_{0} K \tau_{0}+p_{K}, & E C_{K}^{2}=\theta_{K}+\beta_{K}, \\
D_{K}=-a_{K} \sin \omega_{0} K \tau_{0}+b_{K} \cos \omega_{0} K \tau_{0}+q_{K}, & E D_{K}^{2}=\theta_{K}+\beta_{K} .
\end{array}
$$

The mixed covariances are as follows:

$$
\begin{aligned}
& E A_{K} B_{K}=E C_{K} D_{K}=0, \\
& E A_{K} C_{K}=\theta_{K} \cos \omega_{0} K \tau_{0}+\gamma_{K}, \\
& E A_{K} D_{K}=-\theta_{K} \sin \omega_{0} K \tau_{0}+\epsilon_{K}, \\
& E B_{K} C_{K}=\theta_{K} \sin \omega_{0} K \tau_{0}-\epsilon_{K}, \\
& E B_{K} D_{K}=\theta_{K} \cos \omega_{0} K \tau_{0}+\gamma_{K} .
\end{aligned}
$$

Finally every amplitude at frequency $\pi j / T$ is uncorrelated with every amplitude at frequency $\pi K / T, j \neq K$. We are now ready to obtain the likelihood function of the first $4 n$ amplitudes $A_{K}, B_{K}, C_{K}, D_{K}$, for $K=1,2, \cdots, n$. Clearly this is the product of the likelihood function for each quadruple $\left(A_{K}, B_{K}, C_{K}, D_{K}\right)$. First we invert the $4 \times 4$ moment matrix of $\left(A_{K}, B_{K}, C_{K}, D_{K}\right)$. The moment matrix is

$\left(\begin{array}{cccc}\theta_{K}+\alpha_{K} & 0 & \theta_{K} \cos \omega_{0} K \tau_{0}+\tau_{K} & -\theta_{K} \sin \omega_{0} K \tau_{0}+\epsilon_{K} \\ 0 & \theta_{K}+\alpha_{K} & \theta_{K} \sin \omega_{0} K \tau_{0}-\epsilon_{K} & \theta_{K} \cos \omega_{0} K \tau_{0}+\gamma_{K} \\ \theta_{K} \cos \omega_{0} K \tau_{0}+\gamma_{K} & \theta_{K} \sin \omega_{0} K \tau_{0}-\epsilon_{K} & \theta_{K}+\beta_{K} & 0 \\ -\theta_{K} \sin \omega_{0} K \tau_{0}+\epsilon_{K} & \theta_{K} \cos \omega_{0} K \tau_{0}+\gamma_{K} & 0 & \theta_{K}+\beta_{K}\end{array}\right)$

The moment matrix can be inverted by the direct method of calculating the sixteen cofactors. A much simpler method is to partition the matrix into four $2 \times 2$ matrices and invert [5, p. 112]. The determinant of the matrix, denoted by $\lambda_{K}^{2}$, is given by

$$
\lambda_{K}^{2}=\left(\theta_{K}+\alpha_{K}\right)\left(\theta_{K}+\beta_{K}\right)-\left(\theta_{K} \cos \omega_{0} K \tau_{0}+\gamma_{K}\right)^{2}-\left(-\theta_{K} \sin \omega_{0} K \tau_{0}+\epsilon_{K}\right)^{2} .
$$


The inverse of the moment matrix becomes

$\left(\begin{array}{cccc}\frac{\theta_{K}+\beta_{K}}{\lambda_{K}} & 0 & \frac{-\theta_{K} \cos \omega_{0} K \tau_{0}-\gamma_{K}}{\lambda_{K}} & \frac{{ }^{\prime} \theta_{K} \sin \omega_{0} K \tau_{0}-\epsilon_{K}}{\lambda_{K}} \\ 0 & \frac{\theta_{K}+\beta_{K}}{\lambda_{K}} & \frac{-\theta_{K} \sin \omega_{0} K \tau_{0}+\epsilon_{K}}{\lambda_{K}} & \frac{-\theta_{K} \cos \omega_{0} K \tau_{0}-\gamma_{K}}{\lambda_{K}} \\ \frac{-\theta_{K} \cos \omega_{0} K \tau_{0}-\gamma_{K}}{\lambda_{K}} & \frac{-\theta_{K} \sin \omega_{0} K \tau_{0}+\epsilon_{K}}{\lambda_{K}} & \frac{\theta_{K}+\alpha_{K}}{\lambda_{K}} & 0 \\ \frac{\theta_{K} \sin \omega_{0} K \tau_{0}-\epsilon_{K}}{\lambda_{K}} \frac{-\theta_{K} \cos \omega_{0} K \tau_{0}-\gamma_{K}}{\lambda_{K}} & 0 & \frac{\theta_{K}+\alpha_{K}}{\lambda_{K}}\end{array}\right)$

Denoting by $f_{K}\left(A_{K}, B_{K}, C_{K}, D_{K}\right)$ the joint probability density of $\left(A_{K}, B_{K}, C_{K}, D_{K}\right)$, we see $[6, p .311]$ that if we denote moreover by $g_{n}$ the joint probability density of the $4 n$ Gaussian variables $A_{K}, B_{K}, C_{K}, D_{K}$ with $K=1,2, \cdots, n$, that

$$
g_{n}=f_{1}\left(A_{1}, B_{1}, C_{1}, D_{1}\right) f_{2}\left(A_{2}, B_{2}, C_{2}, D_{2}\right) \cdots f_{n}\left(A_{n}, B_{n}, C_{n}, D_{n}\right),
$$

and we see that

$$
\begin{aligned}
g_{n}=(2 \pi)^{-2 n} \prod_{K=1}^{n} \lambda_{K}^{-1} \exp -\frac{1}{2} \sum_{K=1}^{n} \frac{1}{\lambda_{K}}\left\{\left(\theta_{K}+\beta_{K}\right)\left(A_{K}^{2}+B_{K}^{2}\right)\right. \\
+\left(\theta_{K}+\alpha_{K}\right)\left(C_{K}^{2}+D_{K}^{2}\right)-2\left(\theta_{K} \cos \omega_{0} K \tau_{0}+\gamma_{K}\right) A_{K} C_{K} \\
-2\left(-\theta_{K} \sin \omega_{0} K \tau_{0}+\epsilon_{K}\right) A_{K} D_{K}+2\left(-\theta_{K} \sin \omega_{0} K \tau_{0}+\epsilon_{K}\right) B_{K} C_{K} \\
\left.-2\left(\theta_{K} \cos \omega_{0} K \tau_{0}+\gamma_{K}\right) B_{K} D_{K}\right\} .
\end{aligned}
$$

We are now able to compute the lower bound for all unbiased estimates of $\tau_{0}$. In the case of the multi-variate Gaussian distribution the necessary regularity conditions (loc. cit. p. 479) are satisfied, so that we can write

$$
E\left(\tau_{0}^{\prime}-\tau_{0}\right)^{2} \geq\left[E\left(\frac{\partial}{\partial \tau_{0}} \ln g_{n}\right)^{2}\right]^{-1}
$$

For the problem considered here, we establish the fact that

$$
E\left(\frac{\partial}{\partial \tau_{0}} \ln g_{n}\right)^{2}=-E\left(\frac{\partial^{2}}{\partial \tau_{0}^{2}} \ln g_{n}\right),
$$

and since the latter expected value is easier to calculate, we use the form

$$
E\left(\tau_{0}^{\prime}-\tau_{0}\right)^{2} \geq\left[-E \frac{\partial^{2} \ln g_{n}}{\partial \tau_{0}^{2}}\right]^{-1}
$$

Equation (3.8a) is established in the following manner. Consider the identity

$$
E\left(\frac{\partial^{2}}{\partial \tau_{0}^{2}} \ln g_{n}\right) \equiv E\left(\frac{1}{g_{n}} \frac{\partial^{2} g_{n}}{\partial \tau_{0}^{2}}\right)-E\left(\frac{\partial \ln g_{n}}{\partial \tau_{0}}\right)^{2} .
$$

Consider also the identity

$$
E\left(\frac{1}{g_{n}} \frac{\partial^{2} g_{n}}{\partial \tau_{0}^{2}}\right) \equiv \frac{\partial}{\partial \tau_{0}} E\left(\frac{\partial \ln g_{n}}{\partial \tau_{0}}\right)
$$


Hence (3.8a) is established if we show that $E\left(\partial \ln g_{n} / \partial \tau_{0}\right)$ vanishes identically in $\tau_{0}$. The reader can convince himself that $E\left(\partial \ln g_{n} / \partial \tau_{0}\right)$ vanishes identically. Hence (3.8a) is established.

From (3.7) we have

$$
\begin{aligned}
\frac{\partial^{2}}{\partial \tau_{0}^{2}} \ln g_{n}=-\sum_{i=1}^{n} \frac{\partial^{2}}{\partial \tau_{0}^{2}} \ln \lambda_{i} & -\frac{1}{2} \sum_{i=1}^{n}\left\{\left(A_{i}^{2}+B_{i}^{2}\right) \frac{\partial^{2}}{\partial \tau_{0}^{2}}\left(\frac{\theta_{i}+\beta_{i}}{\lambda_{i}}\right)\right. \\
& +\left(C_{i}^{2}+D_{i}^{2}\right) \frac{\partial^{2}}{\partial \tau_{0}^{2}}\left(\frac{\theta_{i}+\alpha_{i}}{\lambda_{i}}\right)-2 A_{i} C_{i} \frac{\partial^{2}}{\partial \tau_{0}^{2}}\left(\frac{\theta_{i} \cos \omega_{0} i \tau_{0}+\gamma_{i}}{\lambda_{1}}\right) \\
& -2 A_{i} D_{i} \frac{\partial^{2}}{\partial \tau_{0}^{2}}\left(\frac{-\theta_{i} \sin \omega_{0} i \tau_{0}+\epsilon_{i}}{\lambda_{i}}\right) \\
& +2 B_{i} C_{i} \frac{\partial^{2}}{\partial \tau_{0}^{2}}\left(\frac{-\theta_{i} \sin \omega_{0 i} \tau_{0}+\epsilon_{i}}{\lambda_{i}}\right) \\
& -2 B_{i} D_{i} \frac{\partial^{2}}{\partial \tau_{0}^{2}}\left(\frac{\theta_{i} \cos \omega_{0 i} \tau_{0}+\gamma_{i}}{\lambda_{i}}\right) .
\end{aligned}
$$

Taking the expected value of each side of the above equation and performing a tedious reduction, we arrive finally at a fairly simple expression. To emphasize the simplicity we define the following quantities:

$$
\begin{array}{ll}
p_{i}=\theta_{i} \cos \omega_{0} i \tau_{0}+\gamma_{i}, & q_{i}=-\theta_{i} \sin \omega_{0} i \tau_{0}+\epsilon_{i}, \\
p_{i}^{\prime}=\frac{\partial p_{i}}{\partial \tau_{0}}, & q_{i}^{\prime}=\frac{\partial q_{i} .}{\partial \tau_{0}}
\end{array}
$$

In terms of these quantities, $(3.8 \mathrm{a})$ becomes

$$
\frac{1}{E\left(\tau_{0}^{\prime}-\tau_{0}\right)^{2}} \leq \sum_{i=1}^{n} \frac{2}{\lambda_{i}}\left\{\left(p_{i}^{\prime 2}+q_{i}^{\prime 2}\right)+\frac{2}{\lambda_{i}}\left(p_{i} p_{i}^{\prime}+q_{i} q_{i}^{\prime}\right)^{2}\right\} .
$$

Finally we reduce this to a form involving the basic covariances and frequencies. We have

$$
\begin{gathered}
p_{i}^{\prime 2}+q_{i}^{\prime 2}=\omega_{0}^{2} i^{2} \theta_{i}^{2}, \\
p_{i} p_{i}^{\prime}+q_{i} q_{i}^{\prime}=-\omega_{0} i \theta_{i}\left(\gamma_{i} \sin \omega_{0} i \tau_{0}+\epsilon_{i} \cos \omega_{0} i \tau_{0}\right) .
\end{gathered}
$$

From (3.6) we obtain

$$
\lambda_{i}=\theta_{i}\left(\alpha_{i}+\beta_{i}-2 \gamma_{i} \cos \omega_{0} i \tau_{0}+2 \epsilon_{i} \sin \omega_{0} i \tau_{0}\right)+\alpha_{i} \beta_{i}-\gamma_{i}^{2}-\epsilon_{i}^{2} .
$$

Denoting by $\omega_{i}$ the frequency $\omega_{0} i$, we obtain

$$
\begin{aligned}
\frac{1}{E\left(\tau_{0}^{\prime}-\tau_{0}\right)^{2}} & \leq 2 \sum_{i=1}^{n} \frac{\omega_{i}^{2} \theta_{i}^{2}}{\theta_{i}\left(\alpha_{i}+\beta_{i}-2 \gamma_{i} \cos \omega_{i} \tau_{0}+2 \epsilon_{i} \sin \omega_{i} \tau_{0}\right)+\alpha_{i} \beta_{i}-\gamma_{i}^{2}-\epsilon_{i}^{2}} \\
+4 & \sum_{i=1}^{n}\left\{\frac{\omega_{i} \theta_{i}\left(\gamma_{i} \sin \omega_{i} \tau_{0}+\epsilon_{i} \cos \omega_{i} \tau_{0}\right)}{\theta_{i}\left(\alpha_{i}+\beta_{i}-2 \gamma_{i} \cos \omega_{i} \tau_{0}+2 \epsilon_{i} \sin \omega_{i} \tau_{0}\right)+\alpha_{i} \beta_{i}-\gamma_{i}^{2}-\epsilon_{i}^{2}}\right\}^{2} .
\end{aligned}
$$

A glance at (3.8c) shows that the value of the right-hand side is independent of any linear pre-detection filtering performed common to both channels. Hence the theoretical 
lower bound is independent of the bandwidth of the receivers in the two channels if they have identical amplitude-frequency responses. In this case (3.8c) becomes

$$
\begin{aligned}
\frac{1}{E\left(\tau_{0}^{\prime}-\tau_{0}\right)^{2}} & \leq 2 \sum_{i=1}^{\infty} \frac{\omega_{i}^{2} \theta_{i}^{2}}{\theta_{i}\left(\alpha_{i}+\beta_{i}-2 \gamma_{i} \cos \omega_{i} \tau_{0}+2 \epsilon_{i} \sin \omega_{i} \tau_{0}\right)+\alpha_{i} \beta_{i}-\gamma_{i}^{2}-\epsilon_{i}^{2}} \\
+ & 4 \sum_{i=1}^{\infty}\left\{\frac{\omega_{i} \theta_{i}\left(\gamma_{i} \sin \omega_{i} \tau_{0}+\epsilon_{i} \cos \omega_{i} \tau_{0}\right)}{\theta_{i}\left(\alpha_{i}+\beta_{i}-2 \gamma_{i} \cos \omega_{i} \tau_{0}+2 \epsilon_{i} \sin \omega_{i} \tau_{0}\right)+\alpha_{i} \beta_{i}-\gamma_{i}^{2}-\epsilon_{i}^{2}}\right\}^{2} .
\end{aligned}
$$

Although the theoretical lower bound is given by $(3.9 \mathrm{a})$, we shall see later that this bound is never attainable by any practical system because of limitations of bandwidth. However, it will be possible to approximate the lower bound given by summing from $i=N_{1}$ to $i=N_{2}$, where $N_{2}-N_{1}=[2 T W], W$ being the receiver bandwidth, and $[x]$ denoting the largest positive integer less than or equal to $x$. Finally we note that divergence of the right-hand side above implies that the lower bound is zero and hence that any estimate attaining this bound converges in mean square to the true value $\tau_{0}$. Later we shall see that for large values of the time-bandwidth product $T W$, the variance of the maximum likelihood estimate approximates the lower bound.

IV. Performance of the finite time correlator. Although there is an extensive literature on correlation methods, there appears to be no discussion in the literatureat least for random signals - of the correlator that maximizes the output signal-to-noise ratio. Hence we give a brief exposition of this here. In order to facilitate a comparison of output signal-to-noise ratio with the lower bound of (3.9), we use the expansions (3.5a) and (3.5b). If we did not wish to make the comparison, it would be more natural to expand in terms of radian frequencies $2 \pi n / T$ instead of the set $\pi n / T$ that was required to obtain the lower bound. Since the Gaussian processes $X_{1}(t)$ and $X_{2}(t)$ possess the same multi-variate distributions as $x_{1}(t)$ and $x_{2}(t)$, we will obtain the correct value for the maximum output signal-to-noise ratio. The following is a block diagram of a correlator:

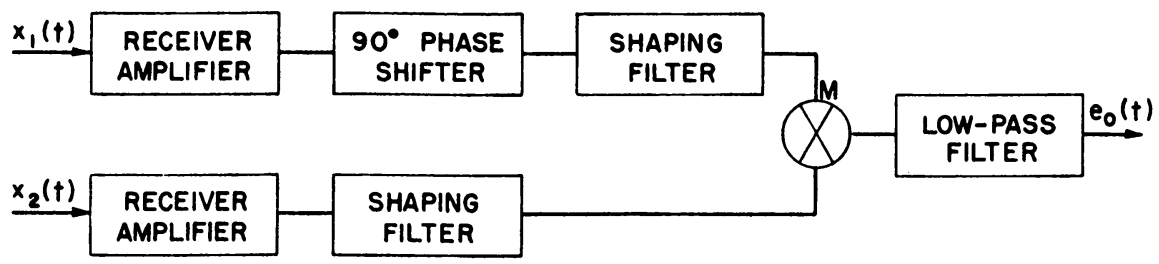

In some correlators the $90^{\circ}$ phase difference is obtained by using a differentiator in one channel, but this merely contributes a factor to the amplitude-frequency response of the shaping filter. To summarize, the phase of the signal in one channel is shifted $90^{\circ}$ - at each frequency - with respect to the other signal, each signal is filtered in the same manner by shaping filters, then multiplied together and low-passed to yield the output $e_{0}(t)$. We shall determine the optimum pre-detection and post-detection filtering in order to maximize the output signal-to-noise ratio. Since we observe $X_{1}(t)$ and $X_{2}(t)$ only during the time interval $0 \leq t \leq T$, it will turn out that the optimum form of postdetection filtering is finite time averaging of the multiplier output. Hence we merely have to determine the proper form of pre-detection filtering.

If we denote by $Y_{2}(t)$ the process obtained by shifting the phase of each frequency component of $X_{2}(t)$ by $90^{\circ}$, we consider the processes 


$$
\begin{aligned}
& X_{1}(t)=\sum_{K=1}^{\infty}\left(A_{K} \cos \omega_{K} t+B_{K} \sin \omega_{K} t\right) \\
& Y_{2}(t)=\sum_{K=1}^{\infty}\left(-C_{K} \sin \omega_{K} t+D_{K} \cos \omega_{K} t\right)
\end{aligned}
$$

for $0 \leq t \leq T$. Since we are interested only in the amplitude $v s$. frequency response of the shaping filter, for convenience we include the effect of this filtering in the $A_{K}, B_{K}$, $C_{K}$, and $D_{K}$. Denoting the amplitude response at frequency $\omega_{K}$ by $f_{K}^{1 / 2}$, we have

$$
\begin{aligned}
& A_{K}=\left(f_{K}\right)^{1 / 2}\left(a_{K}+u_{K}\right), \quad B_{K}=\left(f_{K}\right)^{1 / 2}\left(b_{K}+v_{K}\right), \\
& C_{K}=\left(f_{K}\right)^{1 / 2}\left(a_{K} \cos \omega_{K} \tau_{0}+b_{K} \sin \omega_{K} \tau_{0}+p_{K}\right), \\
& D_{K}=\left(f_{K}\right)^{1 / 2}\left(-a_{K} \sin \omega_{K} \tau_{0}+b_{K} \cos \omega_{K} \tau_{0}+q_{K}\right),
\end{aligned}
$$

where the $a_{K}, b_{K}, u_{K}, v_{K}, p_{K}, q_{K}$ are the random variables defined before in describing the processes $X_{1}(t)$ and $X_{2}(t)$. Clearly we have

$$
\begin{array}{rlrl}
e_{0}=\int_{0}^{T} X_{1}(t) Y_{2}(t) d t & =\sum_{i=1}^{\infty} \sum_{i=1}^{\infty}\left(\alpha_{i j} A_{i} C_{i}+\beta_{i j} B_{i} C_{i}+\gamma_{i j} A_{i} D_{i}+\epsilon_{i j} B_{i} D_{i}\right), \\
\alpha_{i j} & =0, & & i+j \text { even, } \\
& =\frac{2 \omega_{i}}{\omega_{i}^{2}-\omega_{i}^{2}}, & i+j \text { odd }, \\
\beta_{i j} & =0, & i \neq j & \\
& =-T / 2, & i=j \\
\gamma_{i j} & =0, & i \neq j \\
& =T / 2, & i=j \\
\epsilon_{i j} & =0, & & i+j \text { even } \\
& =\frac{2 \omega_{i}}{\omega_{i}^{2}-\omega_{i}^{2}}, & i+j \text { odd } .
\end{array}
$$

Hence we see that

$$
\begin{aligned}
E e_{0} & =\frac{T}{2} \sum_{i=1}^{\infty} E\left(-B_{i} C_{i}+A_{i} D_{i}\right) \\
& =-T \sum_{i=1}^{\infty} f_{i}\left(\theta_{i} \sin \omega_{i} \tau_{0}-\epsilon_{i}\right)
\end{aligned}
$$

To obtain the output noise we first determine $E e_{0}^{2}-\left(E e_{0}\right)^{2}$. We use the following identity valid for Gaussian variables each having mean value zero:

$$
E\left(X_{1} X_{2} X_{3} X_{4}\right)=E X_{1} X_{2} \cdot E X_{3} X_{4}+E X_{1} X_{3} \cdot E X_{2} X_{4}+E X_{1} X_{4} \cdot E X_{2} X_{3} .
$$

Making use of the fact that any two amplitudes with different subscripts are uncorrelated and using the values of $\alpha_{i j}, \beta_{i j}, \gamma_{i j}$, and $\epsilon_{i j}$, we obtain after a tedious calculation 


$$
\begin{aligned}
E e_{0}^{2}-\left(E e_{0}\right)^{2}= & \frac{T^{2}}{2} \sum_{i=1}^{\infty} f_{i}^{2}\left\{2 \theta_{i}^{2} \sin ^{2} \omega_{i} \tau_{0}\right. \\
& \left.+\theta_{i}\left(\alpha_{i}+\beta_{i}-2 \epsilon_{i} \sin \omega_{i} \tau_{0}-2 \gamma_{i} \cos \omega_{i} \tau_{0}\right)+\alpha_{i} \beta_{i}+\epsilon_{i}^{2}-\gamma_{i}^{2}\right\} \\
& +\sum_{\substack{i=1 \\
i+j}} \sum_{i=1} \frac{4 f_{i} f_{i}}{\left(\omega_{i}^{2}-\omega_{i}^{2}\right)^{2}}\left\{\left(\omega_{i}^{2}+\omega_{i}^{2}\right)\left(\theta_{i}+\alpha_{i}\right)\left(\theta_{i}+\beta_{i}\right)\right. \\
& -2 \omega_{i} \omega_{j}\left(\theta_{i} \cos \omega_{i} \tau_{0}+\gamma_{i}\right)\left(\theta_{i} \cos \omega_{i} \tau_{0}+\gamma_{i}\right) \\
& \left.+2 \omega_{i}^{2}\left(\theta_{i} \sin \omega_{i} \tau_{0}-\epsilon_{i}\right)\left(\theta_{i} \sin \omega_{j} \tau_{0}-\epsilon_{j}\right)\right\} .
\end{aligned}
$$

Considering equation (4.2) we see that when $\rho_{12}(-\tau) \neq \rho_{12}(\tau)$, or equivalently that $\epsilon_{i} \neq 0$ for at least one $i$, that a portion of the d-c power is not useful signal. From (4.2) we write

$$
\left(E e_{0}\right)^{2}=\left(\sum_{i=1}^{\infty} \dot{f}_{i} \theta_{i} \sin \omega_{i} \tau_{0}\right)^{2}+\left(\sum_{i=1}^{\infty} f_{i} \epsilon_{i}\right)^{2}-\sum_{i=1}^{\infty} \sum_{i=1}^{\infty} f_{i} f_{i}\left(\theta_{i} \epsilon_{i} \sin \omega_{i} \tau_{0}+\theta_{i} \epsilon_{i} \sin \omega_{i} \tau_{0}\right) .
$$

Only the first term above represents useful signal power. Hence to determine noise power we must add the remaining terms. We denote by $P_{s 0} / P_{n 0}$ the output signal-tonoise ratio. We have

$$
\begin{aligned}
P_{\text {。0 }}=\left[T \sum_{i=1}^{\infty} f_{i} \theta_{i} \sin \omega_{i} \tau_{0}\right]^{2}, \\
P_{n 0}=\frac{T^{2}}{2} \sum_{i=1}^{\infty} f_{i}\left\{2 \theta_{i}^{2} \sin ^{2} \omega_{i} \tau_{0}\right. \\
\left.\quad+\theta_{i}\left(\alpha_{i}+\beta_{i}-2 \epsilon_{i} \sin \omega_{i} \tau_{0}-2 \gamma_{i} \cos \omega_{i} \tau_{0}\right)+\alpha_{i} \beta_{i}+\epsilon_{i}^{2}-\gamma_{i}^{2}\right\} \\
+T^{2}\left(\sum_{i=1}^{\infty} f_{i} \epsilon_{i}\right)^{2}-T^{2} \sum_{i=1}^{\infty} \sum_{i=1}^{\infty} f_{i} f_{i}\left(\theta_{i} \epsilon_{i} \sin \omega_{i} \tau_{0}+\theta_{i} \epsilon_{i} \sin \omega_{i} \tau_{0}\right) \\
+\sum_{\substack{i=1 \\
i+j}}^{\infty} \sum_{i=1}^{\infty} \frac{4 f_{i} f_{i}}{\left(\omega_{i}^{2}-\omega_{i}^{2}\right)^{2}}\{\cdots\} .
\end{aligned}
$$

The optimum correlator is determined by choosing the $\left\{f_{i}\right\}, i=1,2, \cdots$; i.e., the amplitude frequency response of the pre-detection filter, so as to maximize $P_{s 0} / P_{n 0}$, the output signal-to-noise ratio. Theoretically this is accomplished in the following manner. Since $P_{n 0}$ is clearly a positive definite quadratic form in the $\left\{f_{i}, f_{i}\right\}$, there exist orthogonal transformations [7, p. 13] that convert $P_{n 0}$ to diagonal form; namely, $\sum_{i=1}^{\infty} \beta_{i}^{2} f_{i}^{2}$. Since $P_{\text {so }}$ is the square of a linear form, it is converted by a linear transformation to the square of a linear form; namely, $\left(\sum_{i=1}^{\infty} \alpha_{i} f_{i}\right)^{2}$. Hence

$$
P_{s 0} / P_{n 0}=\left(\sum_{i=1}^{\infty} \alpha_{i} f_{i}\right)^{2}\left[\sum_{i=1}^{\infty} \beta_{i}^{2} f_{i}^{2}\right]^{-1}
$$

Applying Schwarz' inequality to the above expression, we write

$$
P_{s 0} / P_{n 0}=\left(\sum_{i=1}^{\infty} \alpha_{i} f_{i}\right)^{2}\left[\sum_{i=1} \beta_{i} f_{i}^{2}\right]^{-1} \leq \sum_{i=1}^{\infty} \alpha_{i}^{2} / \beta_{i}^{2}
$$

Equality is attained in (4.5a) if and only if

$$
f_{i}=K \alpha_{i} / \beta_{i}^{2} \text {. }
$$


Hence (4.5b) yields the form of pre-detection filtering that maximizes the output signalto-noise ratio. However, since $f_{i} \geq 0$ for all $i$, we see that the filter is physically realizable if and only if $\alpha_{i}>0$ or $\alpha_{i}<0$ for all $i$. If this is not the case, the maximum value of $P_{s 0} / P_{n 0}$ attainable is less than the value $\sum_{i=1}^{\infty} \alpha_{i}^{2} / \beta_{i}^{2}$.

V. Comparison of optimum and correlation methods. It appears to be difficult to make a general comparison of the extent to which the best correlator fails in having the optimum performance given by (3.9a). However, we can obtain some insight into the problem by considering a special case; namely, the situation arising when the noise cross-covariance function is symmetric; i.e., $\rho_{12}(-\tau) \equiv \rho_{12}(\tau)$. First we show that for the practical situation in which $\tau_{0}$ is small that $\tau_{0}^{2} / E\left(\tau_{0}^{\prime}-\tau_{0}\right)^{2}$ is identical with the output signal-to-noise ratio. For small values of $\omega_{u} \tau_{0}$ (where $\omega_{u}$ is the highest frequency in the receiver pass band), we see from (4.4a) that

$$
P_{.0} \sim \tau_{0}^{2} T^{2}\left(\sum_{i=1}^{\infty} f_{i} \theta_{i} \omega_{i}\right)^{2}=K^{2} \tau_{0}^{2} .
$$

Moreover, $e_{0}=K \tau^{\prime}$, where $\tau^{\prime}$ is an unbiased estimate of $\tau_{0}$. Then

$$
P_{n 0}=E e_{0}^{2}-\left(E e_{0}\right)^{2}=K^{2} E \tau^{\prime 2}-K^{2} \tau^{2}=K^{2} E\left(\tau^{\prime}-\tau_{0}\right)^{2} \text {. }
$$

Hence,

$$
\frac{P_{00}}{P_{n 0}} \sim \frac{\tau_{0}^{2}}{E\left(\tau^{\prime}-\tau_{0}\right)^{2}}=\left[E\left(\frac{\tau^{\prime}-\tau_{0}}{\tau_{0}}\right)^{2}\right]^{-1}
$$

Thus for small values of $\omega_{\Downarrow} \tau_{0}$, the output signal-to-noise ratio is approximately the reciprocal of the normalized error variance of $\tau^{\prime}$.

Considering now (4.5a), we see that

$$
\alpha_{i}^{2} \sim T^{2} \tau_{0}^{2} \theta_{i}^{2} \omega_{i}^{2} .
$$

If now we approximate to $P_{n 0}$ by considering only the terms proportional to $T^{2}$, we have

$$
\beta_{i}^{2} \sim \frac{T^{2}}{2}\left\{2 \theta_{i}^{2} \sin ^{2} \omega_{i} \tau_{0}+\theta_{i}\left(\alpha_{i}+\beta_{i}-2 \gamma_{i} \cos \omega_{i} \tau_{0}\right)+\alpha_{i} \beta_{i}-\gamma_{i}^{2}\right\} .
$$

(This value of $\beta_{i}^{2}$ will yield an output signal-to-noise ratio greater than the one actually attained, since the actual $P_{n 0}$ is greater than the approximate one assumed.) Hence we obtain

$$
\frac{P_{n 0}}{P_{s 0}} \sim 2 \sum_{i=1}^{\infty} \frac{\theta_{i}^{2} \omega_{i}^{2}}{2 \theta_{i}^{2} \sin ^{2} \omega_{i} \tau_{0}+\theta_{i}\left(\alpha_{i}+\beta_{i}-2 \gamma_{i} \cos \omega_{i} \tau_{0}\right)+\alpha_{i} \beta_{i}-\gamma_{i}^{2}} .
$$

With the exception of the term $2 \theta_{i}^{2} \sin ^{2} \omega_{i} \tau_{0}$ in the denominator of (5.2), the above expression for $P_{n 0} / P_{\bullet 0}$ coincides with the first term of (3.9). In many cases of interest the first term of (3.9) is the dominant term; for example, for uncorrelated noises$\rho_{12}(\tau) \equiv 0$ for all $\tau$ - the second term in (3.9) vanishes. In those cases and to the extent that the approximation in (5.2) is valid, we can conclude that the output signal-to-noise ratio of the best correlator using finite time averaging as a post-detection filter is for all paractical purposes as great as any system whatsoever.

As an example of another type of correlator, consider one which uses an ideal low-pass filter with cutoff frequency $1 / T$ for post-detection, combined of course with an optimum form of pre-detection filtering. It can be shown by using techniques similar to those used in the study of the finite time correlator that under the same assumptions that yielded 
(5.2), symmetric noise cross-correlation and large values of the time-bandwidth product, the output signal-to-noise ratio is given approximately by

$$
\frac{P_{n 0}}{P_{s 0}} \sim \sum_{i=1}^{\infty} \frac{\omega_{i}^{2} \theta_{i}^{2}}{\theta_{i}\left(\alpha_{i}+\beta_{i}-2 \gamma_{i} \cos \omega_{i} \tau_{0}\right)+\alpha_{i} \beta_{i}-\gamma_{i}^{2}} .
$$

Hence under these conditions the correlator using finite time averaging is $3 d b$ superior to the correlator using an ideal low-pass filter with cutoff frequency $1 / T$.

VI. Considerations of time versus bandwidth. The practical considerations of receiver bandwidth necessitate a close examination of $(3.9 \mathrm{a})$. Since we observe the processes $X_{1}(t)$ and $X_{2}(t)$ over the interval $0 \leq t \leq T$, we obtain contributions to the output signal-to-noise ratio from arbitrarily high frequencies. When we discuss a method of extimating $\tau_{0}$ so as to obtain optimum results, we shall see that the actual implementation requires a receiver with infinite bandwidth and hence is not of practical interest. Before we finally restrict ourselves to the practical case of finite bandwidth $W$, we wish to note an interesting result. An examination of the right-hand side of (3.9a) shows that the infinite series either converges or diverges depending upon the relative rates with which the signal and various noise spectra approach zero as the frequency approaches infinity. Hence we conclude that finite time observation using infinite bandwidth need not result in an infinite output signal-to-noise ratio except under special conditons. On the other hand consider the situation that prevails when the bandwidth is finite and restricted to the range $\left(N_{1} \omega_{0}, N_{2} \omega_{0}\right)$, where $N_{2}-N_{1}=[2 T W]$. We wish to obtain the limiting form of (3.8c) as $T \rightarrow \infty$. Since the interval between adjacent radian frequencies is $\pi / T$, we see that by multiplying and dividing the right-hand side of (3.8c) by $\pi / T$, the sum approaches an integral, and we have for large $T$,

$$
\begin{aligned}
& \frac{1}{E\left(\tau_{0}^{\prime}-\tau_{0}\right)^{2}} \\
& \leq \frac{2 T}{\pi} \int_{N_{2} \omega_{0}}^{N_{2} \omega_{0}} \frac{\omega^{2}[\theta(\omega)]^{2} d \omega}{\theta(\omega)\left[\alpha(\omega)+\beta(\omega)-2 \gamma(\omega) \cos \omega \tau_{0}+2 \epsilon(\omega) \sin \omega \tau_{0}\right]+\alpha(\omega) \beta(\omega)-[\gamma(\omega)]^{2}-[\epsilon(\omega)]^{2}}(6.1) \\
& +\frac{4 T}{\pi} \int_{N_{1} \omega_{0}}^{N_{2} \omega_{0}}\left\{\frac{\omega \theta(\omega)\left[\gamma(\omega) \sin \omega \tau_{0}+\epsilon(\omega) \cos \omega \tau_{0}\right]}{\theta(\omega)\left[\alpha(\omega)+\beta(\omega)-2 \gamma(\omega) \cos \omega \tau_{0}+2 \epsilon(\omega) \sin \omega \tau_{0}\right]+\alpha(\omega) \beta(\omega)-[\gamma(\omega)]^{2}-[\epsilon(\omega)]^{2}}\right\}^{2} d \omega .
\end{aligned}
$$

Hence we arrive at the following conclusion-with the obvious restriction that the tracking pass band includes a positive amount of signal spectral energy. With finite observation time and infinite bandwidth the output signal-to-noise ratio may or may not be infinite in accordance with the statistical character of signal and noise. On the other hand the combination of finite bandwidth and infinite observation time always results in an infinite output signal-to-noise ratio.

VII. Asymptotic properties of the maximum likelihood estimate of $\tau_{0}$ for large values of time-bandwidth product. We emphasize the fact that in this section we restrict the analysis to the practical case of finite bandwidth. In order to accomplish this, we rewrite the lower bound for the variance as

$$
\begin{aligned}
\frac{1}{E\left(\tau_{0}^{\prime}-\tau_{0}\right)^{2}} & \leq 2 \sum_{i=N_{2}}^{i=N_{2}} \frac{\omega_{i}^{2} \theta_{i}^{2}}{\theta_{i}\left(\alpha_{i}+\beta_{i}-2 \gamma_{i} \cos \omega_{i} \tau_{0}+2 \epsilon_{i} \sin \omega_{i} \tau_{0}\right)+\alpha_{i} \beta_{i}-\gamma_{i}^{2}-\epsilon_{i}^{2}} \\
+ & 4 \sum_{i=N_{1}}^{i=N}\left\{\frac{\omega_{i} \theta_{i}\left(\gamma_{i} \sin \omega_{i} \tau_{0}+\epsilon_{i} \cos \omega_{i} \tau_{0}\right)}{\theta_{i}\left(\alpha_{i}+\beta_{i}-2 \gamma_{i} \cos \omega_{i} \tau_{0}+2 \epsilon_{i} \sin \omega_{i} \tau_{0}\right)+\alpha_{i} \beta_{i}-\gamma_{i}^{2}-\epsilon_{i}^{2}}\right\}^{2} .
\end{aligned}
$$


$N_{1}$ and $N_{2}$ correspond to the lower and upper radian frequencies $N_{1} \pi / T$ and $N_{2} \pi / T$, where as before $N_{2}-N_{1}=[2 T W], W$ being the receiver bandwidth in cycles per second.

We consider now an estimate of $\tau_{0}$ which for large values of $2 T W$ has a variance that approaches the right-hand side of (7.1). This is the maximum likelihood estimate introduced by R. A. Fisher (for a discussion see Cramér's book, loc. cit. pp. 498-504). Since the properties of the maximum likelihood estimate follow directly from properties of the likelihood function for the random processes $x_{1}(t)$ and $x_{2}(t)$, we use in lieu of this the likelihood function $g_{n}$ of Eq. (3.7), corresponding to the processes $X_{1}(t)$ and $X_{2}(t)$ defined by (3.5a) and (3.5b). As stated earlier, this is justified since the processes $x_{1}(t)$ and $x_{2}(t)$ possess the same multivariate distributions as $X_{1}(t)$ and $X_{2}(t)$. It follows that the maximum likelihood estimate of $\tau_{0}$ determined from the processes $X_{1}(t)$ and $X_{2}(t)$ will have the same statistical properties as the corresponding estimate determined from $x_{1}(t)$ and $x_{2}(t)$. We emphasize now, however, that since $X_{1}(t)$ and $X_{2}(t)$ are not the processes observed, one is still left with the problem of constructing the estimate from the observed processes $x_{1}(t)$ and $x_{2}(t)$. This question will be considered later.

There is very little material in statistical literature on properties of the maximum likelihood estimate of an unknown parameter in a continuous random process. Grenander has shown [8, p. 255-257] that for a process of generalized Markoff type, the maximum likelihood equation has a root that is consistent and asymptotically efficient. More recently P. Whittle [9] has shown that under certain assumptions on the spectrum of a single Gaussian process, the maximum likelihood equation has a root that possesses the usual optimum properties. We are confronted, however, with estimating a parameter of the joint distribution of two processes. Wald [10] has investigated the asymptotic properties of the maximum likelihood estimate of an unknown parameter of a discrete stochastic process. We are able to use a slight extension of his method to obtain our results. The maximum likelihood equation is given by

$$
\frac{\partial}{\partial \tau_{0}} \ln g_{N}=0
$$

where

$$
N=[2 T W] .
$$

We add another assumption to Assumptions 1-3.

Assumption 4. The power spectral density of the signal, $s(t)$, has only a finite number of zeros in the pass band $N_{1} \pi / T$ to $N_{2} \pi / T$. This assumption is not any practical restriction on the generality of the analysis, and is as a matter of fact stronger than required. We denote by $C_{N}\left(\tau_{0}\right)$ the reciprocal of the right-hand side of (7.1). A sequence $\left\{\tau_{N}^{\prime}\right\},\left(N=1,2, \cdots\right.$, ad inf.) of estimates of $\tau_{0}$ is said to be asymptotically efficient in the wide sense if the mean of $\left[C_{N}\left(\tau_{0}\right)\right]^{1 / 2}\left(\tau_{N}^{\prime}-\tau_{0}\right)$ is zero and the variance of $\left[C_{N}\left(\tau_{0}\right)\right]^{1 / 2}$ $\left(\tau_{N}^{\prime}-\tau_{0}\right)$ is 1 in the limit as $N \rightarrow \infty$. Instead of the joint probability density

$$
g_{N}\left(A_{1}, B_{1}, C_{1}, D_{1}, A_{2}, B_{2}, C_{2}, D_{2}, \cdots, A_{N}, B_{N}, C_{N}, D_{N}\right)
$$

which we have, Wald considers a probability density $p_{N}\left(X_{1}, X_{2}, \cdots, X_{N}, \tau_{0}\right)$ defined for all $\tau_{0}$ in some non-degenerate interval $A$ on the real axis. The following four conditions are assumed to hold:

Condition 1. The derivatives $\partial^{i} p_{N} / \partial \tau_{0}^{i}(i=1,2,3)$ exist for all $\tau_{0}$ in $A$ and for al! samples $\left(x_{1}, x_{2}, \cdots, x_{N}\right)$ except perhaps for a set of measure zero. We have furthermore, 


$$
\int_{-\infty}^{\infty} \cdots \int_{-\infty}^{\infty} \underset{\substack{\tau_{0} \in \mathrm{A} \\ \text { l.u.b. }}}{\infty}\left|\frac{\partial^{i} \partial_{N}}{\partial \tau_{0}^{i}}\right| d x_{1} d x_{2} \cdots d x_{N}<\infty \quad(i=1,2) .
$$

Condition 2. For any $\tau_{0}$ in $A, \lim _{N \rightarrow \infty} C_{N}\left(\tau_{0}\right)=\infty$.

Condition 3. For any $\tau_{0}$ in $A$ the standard deviation of $\partial^{2} \log p_{n} / \partial \tau_{0}^{2}$ divided by the expected value of $\partial^{2} \log p_{n} / \partial \tau_{0}^{2}$ (both computed uder the assumption that $\tau_{0}$ is true) converges to zero as $N \rightarrow \infty$.

Condition 4. There exists a positive $\delta$ such that for any $\tau_{0}$ in $A$ the expression

$$
\frac{1}{C_{N}\left(\tau_{0}\right)} E\left[\operatorname{l.u.b.~}_{\tau_{0}}\left|\frac{\partial^{3} \ln p_{n}\left(x_{1}, x_{2}, \cdots, x_{N}, \tau_{0}^{\prime}\right)}{\partial \tau_{0}^{\prime 3}}\right| / \tau_{0}\right]
$$

is a bounded function of $N$ for $\tau_{0}^{\prime}$ in the interval $\left|\tau_{0}^{\prime}-\tau_{0}\right| \leq \delta$.

Conditions 1 and 4 are clearly satisfied, since $g_{N}\left(A_{1}, B_{1}, C_{1}, D_{1}, \cdots, A_{N}, B_{N}, C_{N}, D_{N}\right)$ is of the form

$$
K_{N}\left(\tau_{0}\right) \exp \left\{-Q_{N}\left(A_{1}, B_{1}, C_{1}, D_{1}, \cdots, A_{N}, B_{N}, C_{N}, D_{N} / \tau_{0}\right)\right\},
$$

where $Q_{N}$ is a positive definite quadratic form in the variables $A_{K}, B_{K}, C_{K}, D_{K}, K=$ $N_{1}, \cdots, N_{2}$. Condition 2 was shown to be satisfied in Sec. VI, since the output signalto-noise ratio approaches infinity as $T \rightarrow \infty$. Condition 3 can be shown to be satisfied in the following manner. First we have

$$
\frac{\partial^{2}}{\partial \tau_{0}^{2}} \ln g_{N}=\sum_{i=N_{1}}^{i=N} \frac{\partial^{2}}{\partial \tau_{0}^{2}} \ln f_{i}\left(A_{i}, B_{i}, C_{i}, D_{i}\right),
$$

where as usual $N_{2}-N_{1}=N$. Since this is a sum of $N$ uncorrelated random variables, we have

$$
E\left(\frac{\partial^{2}}{\partial \tau_{0}^{2}} \ln g_{N}\right)^{2}-\left(E \frac{\partial^{2}}{\partial \tau_{0}^{2}} \ln g_{N}\right)^{2}=\sum_{i=N_{2}}^{i=N_{2}}\left\{E\left(\frac{\partial^{2}}{\partial \tau_{0}^{2}} \ln f_{i}\right)^{2}-\left(E \frac{\partial^{2}}{\partial \tau_{0}^{2}} \ln f_{i}\right)^{2}\right\} \leq N \sigma^{2}
$$

where

$$
\sigma^{2}=\sup _{i}\left\{E\left(\frac{\partial^{2}}{\partial \tau_{0}^{2}} \ln f_{i}\right)^{2}-\left(E \frac{\partial^{2}}{\partial \tau_{0}^{2}} \ln f_{i}\right)^{2}\right\}<\infty .
$$

Moreover we have

$$
E\left(\frac{\partial^{2}}{\partial \tau_{0}^{2}} \ln g_{N}\right) \geq N \delta
$$

where

$$
\delta=\inf _{i} E\left(\frac{\partial^{2}}{\partial \tau_{0}^{2}} \ln f_{i}\right)
$$

From Assumption 4, $\delta>0$. Hence,

$\left\{E\left(\frac{\partial^{2}}{\partial \tau_{0}^{2}} \ln g_{N}\right)^{2}-\left(E \frac{\partial^{2}}{\partial \tau_{0}^{2}} \ln g_{N}\right)^{2}\right\}^{1 / 2}\left[E\left(\frac{\partial^{2}}{\partial \tau_{0}^{2}} \ln g_{N}\right)\right] \leq \sigma \delta^{-1} N^{-1 / 2} \rightarrow \infty \quad$ as $N \rightarrow \infty$.

Since Wald's Conditions 1-4 are satisfied, his analysis is applicable directly to our problem. Then we arrive at the result that Eq. (7.2) has a root that is a consistent estimate of $\tau_{0}$. Furthermore any root of (7.2) that is a consistent estimate of $\tau_{0}$ is also asymptotically efficient in the wide sense. 
We have determined the asymptotic properties of the maximum likelihood estimate by analyzing the joint distribution of the random processes $X_{1}(t)$ and $X_{2}(t)$. In order to determine the maximum likelihood estimate explicitly we require the joint distribution of the observed processes $x_{1}(t)$ and $x_{2}(t)$. The determination of this distribution under the weak Assumptions 1-3 requires the inversion of an $N \times N$ matrix, with $N=$ $[2 T W]$. Since the matrix is nonsingular, this inversion can be accomplished in principle. In order to see the required filtering operations on $x_{1}(t)$ and $x_{2}(t)$ more clearly, we make an additional assumption.

Assumption 5. Each of the covariances $\rho_{s}(\tau), \rho_{1}(\tau)$, and $\rho_{2}(\tau)$ is a periodic function of $\tau$ with period $T$.

Clearly it follows from Assumption 3 that $\rho_{12}(\tau)$ is also periodic with period $T$. As Root and Pitcher have shown (loc. cit., p. 313), it follows then that $s(t), n_{1}(t)$, and $n_{2}(t)$ possess Fourier expansions in $(0, T)$ with pairwise orthogonal amplitudes. We write

$$
\begin{aligned}
& s(t)=\underset{n \rightarrow \infty}{\text { l.i.m. }} \sum_{K=1}^{n}\left[a_{K} \cos \Omega_{0} K t+b_{K} \cos \Omega_{0} K t\right], \\
& n_{1}(t)=\underset{n \rightarrow \infty}{\text { 1.i.m. }} \sum_{K=1}^{n}\left[u_{K} \cos \Omega_{0} K t+v_{K} \cos \Omega_{0} K t\right], \\
& n_{2}(t)=\underset{n \rightarrow \infty}{\text { 1.i.m. }} \sum_{K=1}^{n}\left[p_{K} \cos \Omega_{0} K t+q_{K} \sin \Omega_{0} K t\right],
\end{aligned}
$$

for $0 \leq t \leq T$.

We note that $\Omega_{0}=2 \pi / T$, whereas the processes $S(t), N_{1}(t)$, and $N_{2}(t)$ were expanded into components with frequencies being multiples of $\omega_{0}=\pi / T$. Then we write

$$
\begin{aligned}
& x_{1}(t)=s(t)+n_{1}(t)=\underset{n \rightarrow \infty}{\operatorname{li} . \mathrm{m}} . \sum_{K=1}^{n}\left[A_{K} \cos \Omega_{0} K t+B_{K} \sin \Omega_{0} K t\right], \\
& x_{2}(t)=s\left(t+\tau_{0}\right)+n_{2}(t)=\underset{n \rightarrow \infty}{\text { li.m. }} \sum_{K=1}^{n}\left[C_{K} \cos \Omega_{0} K t+D_{K} \sin \Omega_{0} K t\right],
\end{aligned}
$$

for $0 \leq t \leq T$.

To save useless reiteration we use the same notation for the second moments of $A_{K}, B_{K}, C_{K}, D_{K}$ as we did for the corresponding amplitudes of the processes $X_{1}(t)$ and $X_{2}(t)$. Denoting by $h_{n}\left(A_{1}, B_{1}, C_{1}, D_{1}, \cdots, A_{n}, B_{n}, C_{n}, D_{n}\right)$ the joint probability density of the $A_{K}, B_{K}, C_{K}, D_{K}$, the maximum likelihood equation becomes

$$
\begin{aligned}
\frac{\partial}{\partial \tau_{0}} \ln h_{N}=- & \sum_{i=N_{2}}^{i-N_{2}} \frac{\partial}{\partial \tau_{0}} \ln \lambda_{i}-\frac{1}{2} \frac{\partial}{\partial \tau_{0}} \sum_{i=N_{1}}^{i-N}\left\{\left(A_{i}^{2}+B_{i}^{2}\right) \frac{\left(\theta_{i}+\beta_{i}\right)}{\lambda_{i}}\right. \\
& +\left(C_{i}^{2}+D_{i}^{2}\right) \frac{\left(\theta_{i}+\alpha_{i}\right)}{\lambda_{i}}-2\left(A_{i} C_{i}+B_{i} D_{i}\right) \frac{\left(\theta_{i} \cos \omega_{0} i \tau_{0}+\gamma_{i}\right)}{\lambda_{i}} \\
& \left.-2\left(A_{i} D_{i}-B_{i} C_{i}\right) \frac{\left(-\theta_{i} \sin \Omega_{0} i \tau_{0}+\epsilon_{i}\right)}{\lambda_{i}}\right\}=0 .
\end{aligned}
$$

Let us consider what filtering operations are required to obtain the terms in the likelihood equation. The basic tool required is Parseval's equation for Fourier series. This states that if we have the Fourier series 


$$
\begin{aligned}
& y_{1}(t)=\sum_{i=1}^{\infty}\left(a_{i} \cos \frac{2 \pi i}{T}+b_{i} \sin \frac{2 \pi i}{T}\right), \\
& y_{2}(t)=\sum_{i=1}^{\infty}\left(c_{i} \cos \frac{2 \pi i}{T}+d_{i} \sin \frac{2 \pi i}{T}\right),
\end{aligned}
$$

for $0 \leq t \leq T$, then

$$
\frac{2}{T} \int_{0}^{T} y_{1}(t) y_{2}(t) d t=\sum_{i=1}^{\infty}\left(a_{i} c_{i}+b_{i} d_{i}\right)
$$

Examining the term

$$
\sum_{i=N_{1}}^{i=N_{2}}\left(A_{i}^{2}+B_{i}^{2}\right)\left(\frac{\theta_{i}+\beta_{i}}{\lambda_{i}}\right),
$$

we see that if $x_{1}(t)$ is passed through a linear filter with gain $\left[\left(\theta_{i}+\beta_{i}\right) / \lambda_{i}\right]^{1 / 2}$ at frequency $\Omega_{0} i$, yielding an output $y_{1}(t)$, then

$$
\sum_{i=N_{1}}^{i=N_{2}}\left(A_{i}^{2}+B_{i}^{2}\right)\left(\frac{\theta_{i}+\beta_{i}}{\lambda_{i}}\right)=\frac{2}{T} \int_{0}^{T}\left[y_{1}(t)\right]^{2} d t .
$$

An analogous operation is performed on $x_{2}(t)$ to obtain the term

$$
\sum_{i=N_{1}}^{i=N_{2}}\left(C_{i}^{2}+D_{i}^{2}\right)\left(\frac{\theta_{i}+\alpha_{i}}{\lambda_{i}}\right) \text {. }
$$

To obtain the term

$$
\sum_{i=N_{i}}^{i=N_{i}}\left(A_{i} C_{i}+B_{i} D_{i}\right)\left(\frac{\theta_{i} \cos \Omega_{0} i \tau_{0}+\gamma_{i}}{\lambda_{i}}\right),
$$

it is necessary to pass $x_{1}(t)$ and $x_{2}(t)$ through identical linear filters with gain $\left[\left(\theta_{i} \cos \Omega_{0} i \tau_{0}+\gamma_{i}\right) / \lambda_{i}\right]^{1 / 2}$ obtaining outputs $Z_{1}(t)$ and $Z_{2}(t)$. Then

$$
\sum_{i=N_{1}}^{i=N_{2}}\left(A_{i} C_{i}+B_{i} D_{i}\right)\left(\frac{\theta_{i} \cos \Omega_{0} i \tau_{0}+\gamma_{i}}{\lambda_{i}}\right)=\frac{2}{T} \int_{0}^{T} Z_{1}(t) Z_{2}(t) d t .
$$

In order to obtain the term

$$
\sum_{i=N_{1}}^{i=N}\left(A_{i} D_{i}-B_{i} C_{i}\right)\left(\frac{-\theta_{i} \sin \Omega_{0} i \tau_{0}+\epsilon_{i}}{\lambda_{i}}\right)
$$

we note that we must first pass $x_{2}(t)$ through a $90^{\circ}$ phase shifter, obtaining at the output

$$
x_{2}^{\prime}(t)=\sum_{i=N_{1}}^{i=N_{2}}\left(-C_{i} \sin \Omega_{0} i t+D_{i} \cos \Omega_{0} i t\right) .
$$

Then $x_{1}(t)$ and $x-(t)$ are passed through identical linear filters with gain

$$
\left(\frac{-\theta_{i} \sin \Omega_{i} i \tau_{0}+\epsilon_{i}}{\lambda_{i}}\right)^{1 / 2}
$$

producing processes $e_{1}(t)$ and $e_{2}(t)$. Then

$$
\sum_{i=N_{2}}^{i-N_{2}}\left(A_{i} D_{i}-B_{i} C_{i}\right)\left(\frac{-\theta_{i} \sin \Omega_{0} i \tau_{0}+\epsilon_{i}}{\lambda_{i}}\right)=\frac{2}{T} \int_{0}^{T} e_{1}(t) e_{2}(t) d t .
$$


It is interesting to note that the last term in the likelihood equation (7.5) requires exactly the same operations as the finite time correlator; namely, $90^{\circ}$ phase shifting of one process, linear filtering, multiplication, and finite time averaging.

Brief consideration of Eq. (7.5) shows that it cannot be solved explicitly for $\tau_{0}^{\prime}$ in terms of the various filtered quantities. One possible approach is to evaluate $\ln h_{N}$ for a sequence of values of $\tau_{0}$ and determine its absolute maximum. Clearly this involves a large number of operations in comparison to those required by a simple correlation system. In the next section we discuss a method for obtaining an approximate maximum likelihood estimate.

VII. An approximation to the maximum likelihood estimate. In order to avoid the obvious difficulties in solving (7.5), we discuss an approximation to the maximum likelihood estimate. The method is based upon the fact that in the process of tracking one is always striving to achieve the condition $\tau_{0}=0$. Hence consider $\partial / \partial \tau_{0} \ln h_{N}$ evaluated at $\tau_{0}=0$. This condition prescribes the filtering operations for $x_{1}(t)$ and $x_{2}(t)$ for the "on target" situation. Let us denote by $E_{\tau}\left(\partial / \partial \tau_{0} \ln h_{N}\right)$ the expected value of $\partial / \partial \tau_{0} \ln h_{N}$ computed when $\tau$ is true. We have shown in Sec. III that $E_{\tau_{0}}\left(\partial / \partial \tau_{0} \ln h_{N}\right)=0$. Hence this estimate has no average d-c bias due to noise for the "on target" condition. Moreover, the output signal-to-noise ratio for small deviations from $\tau_{0}=0$ attains the maximum value possible. As the true value of $\tau_{0}$ continues to deviate from zero, the output signal-to-noise ratio deteriorates below the maximum attainable.

It is of practical interest to investigate the "steering pattern" of this approximate estimate; i.e., $E_{\tau}\left(\partial / \partial \tau_{0} \ln h_{N}\right)_{\tau \bullet-0}$. After a few algebraic manipulations, we obtain

$$
E_{\tau}\left(\frac{\partial}{\partial \tau_{0}} \ln h_{N}\right)=\sum_{i=N_{2}}^{i=N} \frac{2 \omega_{i} \theta_{i}^{2}}{\lambda_{i}^{2}}\left\{\left(\lambda_{i}+\epsilon_{i}^{2}\right) \sin \omega_{i} \tau+2 \epsilon_{i}\left(\theta_{i}+\gamma_{i}\right) \sin ^{2} \frac{\omega_{i} \tau}{2}\right\} .
$$

Hence we see that the average d-c output consists of the sum of a symmetric term and an anti-symmetric term. For background noise with symmetric cross-correlation $\left(\epsilon_{i} \equiv 0\right.$ for every $i$ ), the output consists solely of a symmetric term.

\section{REFERENCES}

1. Kari Karhunen, Über lineare Methoden in der Wahrscheinlichkeitsrechnung, Annales Academiae Scientiarum Fennicae, Helsinki, 1947

2. Harold Cramér, On the theory of stationary random processes, Ann. Math. 41, No. 1, (1940)

3. W. L. Root and T. S. Pitcher, On the Fourier series expansion of random functions, Ann. Math. Statistics 26, No. 2, (June 1955)

4. H. B. Mann, Introduction to the theory of stochastic processes depending on a continuous parameter, Natl. Bur. Standards Rept. 1293, May 1951

5. R. A. Frazer, W. J. Duncan, and A. R. Collar, Elementary matrices, Cambridge, University Press, 1950

6. Harold Cramér, Mathematical methods of statistics, Princeton University Press, 1946

7. R. Courant and D. Hilbert, Methoden der mathematischen Physik, Vol. I, Berlin, 1931

8. Ulf Grenander, Stochastic processes and statistical inference, Arkiv för Matematik, Band 1, Häfte 3, Stockholm, 1950

9. P. Whittle, Estimation and Information in Stationary Time Series, Arkiv för Matematik, Band 2, Nr. 23, 1953

10. A. Wald, Asymptotic properties of the maximum likelihood estimate of an unknown parameter of a discrete stochastic process, Ann. Math. Statistics, 19, No. 1, (March 1948) 